\title{
On the nature and order of organizational processing: A reply to Peterson
}

\author{
STEPHEN PALMER and IRVIN ROCK \\ University of California, Berkeley, California
}

\begin{abstract}
Several points in the original article by Palmer and Rock (1994) are clarified in light of Peterson's (1994) commentary. First, we address several issues related to the nature and order of organizational processes in our theory. We argue that many of her objections result from misconceptions about our theory. We attempt to clarify the relevant issues, including the fact that we intended the theory to describe an ordering of processes in cascade rather than as a sequence of classical discrete stages. Second, we discuss Peterson's objections to our discussion of her findings on the relation between figureground processing and object recognition. We argue against her "prefigural recognition" proposal on logical grounds and provide an alternative account of her findings in terms of our theory.
\end{abstract}

We thank Mary Peterson for her thoughtful commentary on our article, "Rethinking perceptual organization: The role of uniform connectedness" (Palmer \& Rock, 1994). She spent considerable time reviewing it prior to publication, as we did on revising it in light of her criticisms. Despite these exchanges, there remain substantial differences between our views on several issues. In this reply, we respond to the remaining criticisms she discusses in her commentary. We hope this final public exchange will serve to clarify some of the controversies and to shed more light on what are certainly very complex theoretical questions concerning the nature of perceptual organization.

\section{Historical Precedent}

Before turning to the substantive theoretical issues raised by Peterson, we must thank her and William Ittelson for pointing out the very relevant passage from Koffka's (1935) book, quoted by Peterson at the end of her commentary. It is obvious that Koffka appreciated not only the problem we raised of designating "entry-level units" of organization prior to perceptual grouping, but its solution in terms of what we call "uniform connectedness" (UC). Needless to say, we would have cited Koffka's famous book had we remembered this particular passage. His discussion provides important historical precedent for the basic idea we discussed: namely, that $\mathrm{UC}$ is a crucial factor in defining the initial units of perceptual organization.

Still, we maintain that the existence of Koffka's brief discussion changes little of substance about the content of our article. It is certainly not the case, for example, that the role of UC in perceptual organization is well appreciated or well understood among modern perceptual

Correspondence should be addressed to S. Palmer, Psychology Department, University of California, Berkeley, CA 94720 (e-mail: palmer@cogsci.berkeley.edu). psychologists. Koffka notwithstanding, UC's role prior to grouping is not discussed in any textbooks on perception or in any research papers with which we are familiar. More importantly, we believe we have gone significantly beyond Koffka's contribution. Although Koffka clearly mentions UC of color (including luminance) as a factor in perceptual organization, he does not discuss it fully nor develop its relation to other processes of perceptual organization. The notion of UC we developed is far more general than this, encompassing texture, motion, and possibly other factors as well. Moreover, we analyze the logical relations that exist between UC and other organizational processes, relations that form the basis for the theoretical approach we developed in our article.

\section{On the Nature and Order of Organizational Processing}

A significant portion of Peterson's commentary is taken up by objections to what she characterizes as our view of organization in terms of a strict sequence of stages. We have two sorts of problems with her criticisms. One is the quite general observation that we did not intend to imply a discrete stage model of processing (e.g., Sternberg, 1969). The other is that we believe several of her specific claims to be inaccurate. We will discuss each of these issues in turn.

Serial stages versus processes in cascade. Peterson appears to have assumed that we are proposing a strict, discrete stage model of processing. It is understandable that she might have done so, since we included a flow diagram consisting of boxes and arrows (Palmer \& Rock, 1994, Figure 13, reproduced here as Figure 1) without any specific disclaimer that this should not be interpreted as a classical discrete stage model. We intended the flowchart of Figure 1 merely to summarize a set of logical constraints that our analysis led us to believe exists among the ordering of the various organizational processes that we discussed. The image has to come first. Basic-level units have to come before grouping 


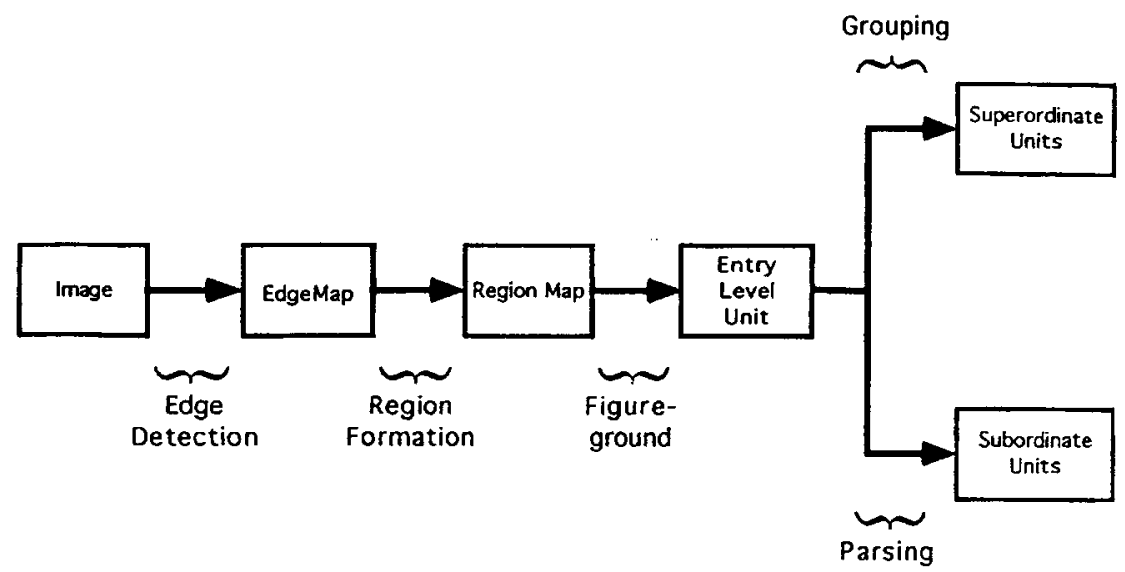

Figure 1. A flowchart representation of the relations among processes proposed to be involved in perceptual organization. From "Rethinking Penceptual Organization: The Role of Uniform Connectedness," by S. Palmer and I. Rock, 1994, Psychonomic Bulletin \& Review, I, p. 42. Copyright 1994 by the Psychonomic Society, Inc. Reprinted by permission.

and parsing, because the latter presuppose units whereas the former does not. Edge detection must come before designation of regions, if regions are defined indirectly by their boundaries. And so forth.

However, we view the diagram as representing an ordered set of processes in cascade rather than as a set of discrete stages: full processing within each box requires a significant amount of time, and partial results are continuously available to the next process so that processing overlaps strongly (McClelland, 1979). We generally tried to refer to "processes" rather than "stages," but rereading the article shows that on several occasions we did use "stage" terminology. This could have been interpreted to mean that we were thinking in terms of classical discrete stages.

To complicate matters further, we suspect a complete theory of perceptual organization will require feedback from later stages to earlier ones. For example, feedback from the region level may influence the identification of edges, reinforcing the extraction of weak edges that are expected from otherwise strongly indicated regions. The net effect of a series of processes in cascade with feedback is that the ordering we proposed can only be used to predict the order in which processes begin, not necessarily the order in which they end. Obviously, this makes it difficult to derive firm predictions from the theory, but it would be foolish to expect a theory of perceptual organization to be simple. Many complex and interrelated factors are clearly at work, and any theory that does justice to their interaction will necessarily be complex.

Parsing and stereopsis. At a more specific level, we believe Peterson has misunderstood the order of processing she attributes to our theory. She characterizes it as a simple linear order of seven organizational processes (see Peterson, 1994, her Table 1), but this does not accurately represent our views. Her ordering of the first four processes is correct, but that of the last three is not, being based on an erroneous inference she made from one of our demonstrations.

The main issue in question is the relative ordering of parsing and stereopsis. ${ }^{.}$On the basis of her analysis of our Figure 8 (her Figure 1), Peterson argues that stereopsis follows parsing in our theory. She first points out that our demonstration indicates that stereopsis operates on units smaller than those defined by uniform connectedness. She then lists two alternative interpretations and mistakenly asserts that we would favor the second: that parsing of UC regions into smaller units must occur before stereopsis.

In fact, however, we endorse the first interpretation: that some process other than UC can provide the elementary units over which stereopsis operates. Indeed, we discussed the nature of the units over which fusion occurs in modern theories of stereopsis quite explicitly in several places (Palmer \& Rock, 1994, pp. 49, 50, and Note 12 on p. 55). All of our discussion is clearly consistent with the assumption that stereo correspondence can work on units smaller than UC regions: the output of individual receptors (e.g., Marr \& Poggio, 1977), edge detectors (e.g., Marr \& Poggio, 1979), or spatial frequency filters (e.g., Jones \& Malik, 1992). In all three cases, these entities clearly exist prior to region formation by UC. Thus, the postparsing option chosen for us by Peterson is incompatible with our explicit statements about stereopsis, and her ordering of the last three processes in her Table 1 is therefore not implied by our theory. It is actually inconsistent with our stated views.

The nature of organizational units. Peterson further argues that the possibility of stereopsis operating on units smaller than UC regions undermines our claim that UC occupies a privileged position in perceptual organization by virtue of defining the primary entry level units. We disagree. Because the nature of our disagreement suggests that Peterson may have misunderstood 
what we meant by "organizational units," we want to clarify this concept.

As we have stated (Palmer \& Rock, 1994, p. 38), we take the units of perceptual organization to refer to entities in the part--whole hierarchy of object perception at different size scales. As examples, we mentioned texture elements, object parts, whole objects, aggregations of many objects, or even complex extended scenes. All of these are what we would call "object candidates": region-based entities in the image that could, in principle, correspond to reasonable perspective views of two- or three-dimensional objects. They also have the property that they can be derived by either unifying or dividing other organizational units in the hierarchy.

We specifically did not include smaller entities, such as pixels and edges, because these are not object candidates: they are zero- and one-dimensional entities (respectively) rather than two-dimensional ones, neither of which could sensibly be taken to correspond to an object in the environment except under highly improbable viewing conditions. In our view, then, representations such as pixels and edges are image-processing entities required for segmenting the image into regions (among other things), but they are not organizational units in the part-whole hierarchy, at least as we discuss it. Therefore, we ague, they logically cannot provide entry into the hierarchy.

Multi-level operation of uniform connectedness. A final issue surrounding Peterson's ordering of processes in her Table 1 concerns her statement that there is a "conundrum" surrounding the possibility that UC might operate at both pre- and postconstancy levels. We do not understand what the conundrum is. The principle of UC can operate at any number of levels within the visual system if it is applied to representations based on different kinds of information. In fact, we simplified matters by saying that UC operates just twice, once at the preconstancy level and once at the postconstancy level. Within the preconstancy phase of processing, for example, we believe it operates on (at least) luminance, color, texture, and motion. It seems straightforward that UC might also operate on each of these properties after depth and other constancy-related processing have modified their representations to arrive at perceived (rather than retinal) values.

\section{On Figure-Ground and Object Recognition}

The remainder of Peterson's commentary criticizes our theory for what she perceives to be its inadequacies in dealing with the relation between figure-ground processing and object recognition. In fact, figure-ground processing was not a major focus of our paper, and we did not talk about object recognition at all except for a single sentence in which we said it was a late process. Even so, we will end our reply by discussing her objections, because they are a major focus of Peterson's commentary.

Peterson and Gibson $(1991,1993)$ have reported convincing evidence that figure-ground organization is influenced by what they call "denotivity": roughly, the ex- tent to which a given region's shape looks like that of a known object type. ${ }^{2}$ All else being equal, regions high in denotivity are more likely to be perceived as figure initially and for longer periods of time than regions low in denotivity. They interpret their findings as showing that object recognition occurs earlier than has generally been thought: specifically, before figure-ground processing. We interpret them as showing that figure-ground organization (or rather its final result) occurs later than has generally been thought. Both possibilities are logically consistent with the fact that denotivity affects figureground perception. How is one to decide between them?

The major difficulty we see with Peterson's proposal that object recognition occurs before figure-ground organization is that object recognition seems logically to imply a comparison between the shape of the object candidate and the shapes of known object types. This, in turn, seems logically to require that figure-ground organization occur prior to object recognition, because a contour imparts shape only to the region on its figural side; the ground is seen to extend uniformly behind the figural region. Such considerations lead us to believe that some distinction between figure and ground processing must occur prior to object recognition. ${ }^{3}$ The logic appears airtight.

But how then is it possible that denotivity (presumably due to object recognition processes) can influence figure-ground perception, as Peterson and Gibson have found? By Peterson's account, there exist "prefigural recognition processes" that operate directly on "edges per se, and not necessarily on the edges of regions already (partially or fully) determined to be figure" (Peterson, 1994). She proposes that these prefigural recognition processes "carve the edges that can be detected early in visual processing into parts from both sides simultaneously, therefore indicating different sets of parts along the edge's two sides" (Peterson, 1994, emphasis hers). These different part sets are then used in parallel to access representations of known object types in memory. The output activations from these recognition units then provide input to figure-ground computations, favoring the sides of edges that correspond to known object types.

The first question is whether Peterson's proposal actually avoids the logical dependency of object recognition on shape (and therefore on figural regions) mentioned above. We do not see how it can. True edges do not have shape; they merely impart shape to the regions they bound. We therefore believe that some region-based figural distinction-primitive, local, and preliminary though it may be-is logically implied in her theory prior to object recognition. In fact, we believe it is hidden in the process of "carving the edges ... into parts from both sides simultaneously." Here is our thinking.

The only method of dividing an object into parts along a contour (that we know of, anyway) is parsing it at concave discontinuities or deep concavities (Hoffman \& Richards, 1985). But this implicitly requires a figureground distinction because an edge per se is neither concave nor convex at an extremum of curvature. It is both: 
concave on one side and convex on the other. Therefore, dividing a contour at a given curvature extremum necessarily implies that the region on the concave side is being taken as figure at that point, at least implicitly.

In Peterson's description, this figural designation is not mentioned as such, but it is implied by her speaking of assigning parsing points along an edge to one side or the other. These sides are parts of regions, not parts of edges. Without this (or some equivalent) distinction between the regions on opposite sides of the edge, each extremum would simply belong to the edge itself, and there would be no way to distinguish between the "different sets of parts along the edge's two sides" (Peterson, 1994). If there were no representation of figural regions in the process Peterson envisions, we do not understand how the various parsing points along an edge could even be unified for matching to representations in memory. The closest we believe Peterson can come to a "prefigural recognition process" would be one based on an implicit mosaic organization in which all regions are initially treated as though they were figural. But this does not sound like what Peterson has in mind.

Within the framework of our own theory, figureground processing must at least begin before object recognition can occur, for all the reasons given above. It cannot have ended prior to object recognition, of course, because that would contradict Peterson and Gibson's findings. For both of these statements to be true, we appeal to the hypothesis that these processes operate in cascade with feedback. That they operate in cascade means that although figure-ground processing must begin before object recognition can take place, it will not necessarily have ended. This allows for the possibility that the logically later process of object recognition can influence the logically earlier process of figure-ground organization through feedback.

Here is how denotivity might affect figure-ground organization within our theory, step by step. For simplicity, let us suppose there are just two regions with a common border: a white one that has a recognizable shape, and a black one that does not. Initially, the operation of UC would provide a mosaic representation consisting of both regions. Simple shape properties, such as size, convexity, surroundedness, symmetry, and so forth, would be computed for all regions simultaneously and used in figure-ground determination. (We call these measures "simple" not because they are necessarily easy to compute, but because, unlike object recognition, they do not depend on accessing memories of specific shapes.) The two regions then would compete for figural status along their shared border, based on some composite of these factors, which we will call "figurality." Early results of this figure-ground competition would then determine the extent to which the shapes of different regions get processed by higher level organizational processes, such as grouping and parsing. (The shape of a nonfigural region at a contour would not get processed simply because the edge does not impart shape to it.) If one region were very highly figural and the other were not figural at all-such as a small, convex, symmetrical white blob surrounded by a big, concave, black background-only the highly figural region would be processed as figure, at least initially.

But if such factors prove equivocal, as they surely would in the well-controlled ambiguous displays Peterson employs in her experiments, neither region would win in the early phases of figural competition. In this event, we assume that both regions would then be treated as figural by later processes, including parsing, grouping, and object recognition. (We are presuming that object recognition follows parsing and grouping because both seem to be necessary for recognition of everyday objects.) If one of the regions strongly activates the representation of a known object type in memory and the other does not, as is typically the case in Peterson's experiments, this information could feed back to the figure-ground process, selectively increasing the corresponding region's figurality. This boost in figurality would cause the high-denotivity region to begin to dominate the low-denotivity region in the figure-ground competition. This domination would then escalate as the competition between adjacent regions continues and as further feedback from the increasingly successful object recognition process further reinforces the figurality of the high-denotivity region. When some threshold level of figurality is exceeded by one of the regions, that region would be consciously perceived as figure and the other as ground. Within this scenario, the high-denotivity region is more likely to be perceived as figure and the low-denotivity region as ground.

We argue that such dynamical mechanisms could operate within the framework of our theory to produce effects of object recognition on figure-ground organization, such as Peterson and her colleagues have reported. ${ }^{4}$ As just described, they would allow high-denotivity regions to be perceived initially as figure with a higher probability than low-denotivity regions. With some additional assumptions about multistability and the stabilizing effect of feedback from the object recognition level, we believe it could also account for perceiving the high-denotivity region for longer periods of time than low-denotivity regions during extended viewing.

Contrary to Peterson's claims, then, it is quite possible for a theory in which figure-ground processing precedes object recognition to account for effects of denotivity on figural perception. Of course, this does not mean that it actually happens this way. To find out, critical tests between alternative theories would have to be devised and carried out.

\section{REFERENCES}

Biederman, I. (1987). Recognition-by-components: A theory of human image understanding. Psychological Review, 94, 115-147. EPSTEIN, W., \& DeShazo, D. (1961). Recency as a function of perceptual oscillation. American Journal of Psychology, 74, 21 5-223. Hoffman, D. D., \& Richards, W. A. (1985). Parts of shape recogni- 
tion. In S. Pinker (Ed.), Visual cognition (pp. 65-96). Cambridge, MA: MIT Press.

JONES, D., \& MALIK, J. (1992). A computational framework for determining stereo correspondence from a set of linear spatial filters. In G. Sandini (Ed.), Proceedings of the Second European Conference on Computer Vision (pp. 395-410). Berlin: SpringerVerlag.

KOFFKA, K. (1935). Principles of Gestalt psychology. New York: Harcourt, Brace.

MARR, D. (1982). Vision. San Francisco: W. H. Freeman.

MARR, D., \& PogGIO, T. (1977). Cooperative computation of stereo disparity. Science, 194, 283-287.

MARR, D., \& PoGgIO, T. (1979). A computational theory of human stereo vision. Proceedings of the Royal Society of London: Series B, 204, 301-328.

MCCLELLAND, J. L. (1979). On the time relations of mental processes: An examination of systems of processes in cascade. Psychological Review, 86, 287-330.

Palmer, S., \& Rock, I. (1994). Rethinking perceptual organization: The role of uniform connectedness. Psychonomic Bulletin \& Review, 1, 29-55.

Peterson, M. A. (1994). The proper placement of uniform connectedness. Psychonomic Bulletin \& Review, 1, 509-514.

Peterson, M. A., \& Gibson, B. S. (1991). The identification of figure-ground relationships: Contributions from shape recognition processes. Bulletin of the Psychonomic Society, 29, 199-202.

Peterson, M. A., \& GiBson, B. S. (1993). Shape recognition contributions to figure-ground organization in three-dimensional displays. Cognitive Psychology, 25, 383-429.

Rock, I. (1975). An introduction to perception. New York: Macmillian. RoCK, I., \& KREMEN, I. (1957). A re-examination of Rubin's figural aftereffect. Journal of Experimental Psychology, 53, 23-30.

STERNBERG, S. (1969). The discovery of processing stages: Extensions of Donders' method. In W. G. Koster (Ed.), Attention and performance II (pp. 276-315). Amsterdam: Elsevier/North-Holland.
WaLlaCh, H. (1949). Some considerations concerning the relation between perception and cognition. Journal of Personality, 18, 6-13.

\section{NOTES}

1. We should note that in emphasizing stereopsis, Peterson focuses on an issue that is actually quite peripheral to our concerns in the article. We were not particularly concerned with depth perception except that, given its occurrence--based on any and all cues-perceptual reorganization occurs in which perceived connectedness may now lead to altered organization of a scene. Stereopsis came up only because it was the relevant depth cue in the demonstration in our (Palmer \& Rock, 1994) Figure 8 (or Peterson's, 1994, Figure 1).

2. It is perhaps worth noting for historical accuracy that the "discovery" of which Peterson speaks in her commentary "that outputs from representations of shapes in memory can contribute to figure-ground organization" was first made by Rubin in 1921. A more recent demonstration was given 20 years ago by Rock $(1975$, p. 358) in which a profile face is always perceived as figure before the region on the opposite side of their common boundary.

3. We are not alone in making this assumption. As Peterson rightly points out, it has been made by virtually all modern perceptual theorists (e.g., Biederman, 1987; Marr, 1982), to which we would add many earler theorists (e.g., Epstein \& DeShazo, 1961; Rock \& Kremen, 1957; Wallach, 1949).

4. Another possibility would be to appeal to a rapid oscillation of organization below the level of awareness (Epstein \& DeShazo, 1961). Each region would be sampled as figure alternately, and the one that constitutes a known object would then be selected for conscious perception as figure. We prefer the explanation in terms of processes in cascade with feedback, but we mention the oscillation hypothesis for the sake of completeness.

(Manuscript received June 13, 1994; revision accepted for publication July 28,1994 .) 